\title{
Arbor
}

\section{Estado actual y perspectivas de la Neurociencia en España}

José López Barneo

Arbor CLXVIII, 662 (Febrero 2001), 223-228 pp.

\section{Introducción}

La Neurociencia se encarga del estudio multidisciplinar e integrado del sistema nervioso, y en su concepción actual es una disciplina relativamente reciente. Los departamentos y programas de doctorado en Neurociencia más influyentes aparecieron en universidades americanas y europeas en la década de los sesenta entre otras causas como reacción al auge y desarrollo de la Bioquímica y la Biología molecular. Se consideró que el abordaje meramente reduccionista de los procesos neurobiológicos podría conducir a la pérdida de la visión global del cerebro, limitando la posibilidad de comprender un órgano cuyas propiedades más genuinas emergen del funcionamiento integrado de sus elementos constitutivos. La Neurociencia nació con una vocación holística aunque, paradójicamente, los mayores avances en este campo se han producido asociados a la Biología molecular, la Fisiología celular o la Farmacología. Esta aparente contradicción que existe entre la perspectiva integradora del estudio del sistema nervioso y el éxito del abordaje metodológico reduccionista ha sido una constante en la evolución de la Neurociencia que posiblemente también determine su evolución futura.

Como es bien conocido, España ha sido en los últimos siglos un país con una actividad científica muy precaria y lo poco que se generó durante el auge intelectual del primer tercio del siglo XX fue destruido por la Guerra Civil y el exilio posterior. Como ocurrió con otras disciplinas, la Neurociencia española moderna se originó prácticamente de novo, por lo que es fácil identificar a las personas e instituciones que tuvieron el «efecto fundador» y dibujar el «árbol genealógico» de 
los neurocientíficos de la actualidad. Aunque por diversas razones, entre otras su naturaleza interdisciplinar, las raices de la Neurociencia española son más borrosas y menos definidas que las de otras ciencias biomédicas, es indudable que el desarrollo actual se inició gracias al trabajo de unos pocos profesores e investigadores y se debe en gran medida a la influencia que éstos ejercieron sobre la generación posterior. El objetivo de este breve recorrido por la Neurociencia española no es describir la labor o los logros de personas y grupos concretos sino resaltar los hechos y situaciones que han tenido mayor trascendencia. Este artículo refleja una opinión personal espontánea sobre el estado actual y perspectivas de la Neurociencia española que indudablemente está influenciada por la cercanía del autor a determinadas personas e instituciones. Espero que este sesgo no reste valor conceptual a la visión de conjunto.

\section{El pasado reciente y la situación actual}

Los primeros balbuceos de la Neurociencia española están ligados a la actividad científica de grupos en la Universidad, generalmente dentro de departamentos de Fisiología o Anatomía. La Neurociencia nunca creció con la estructura administrativa universitaria debido a que no han existido, ni existen, en España cátedras o departamentos universitarios de Neurociencia y, salvo alguna excepción muy puntual, tampoco se han impartido asignaturas troncales con esa denominación. Como es bien conocido la escuela de Cajal desapareció casi completamente por lo que no tuvo una influencia decisiva en el resurgir de la Neurociencia española. El instituto Cajal del Consejo Superior de Investigaciones Científicas (CSIC), heredero en parte del Instituto de Investigaciones Biológicas creado por Cajal, contó con algún investigador en Neurohistología y Neurofisiología de nivel internacional, pero en su conjunto fue durante mucho tiempo una reliquia del pasado. Aunque en la decada de los sesenta y principio de los setenta ya existía un núcleo estable de neurocientíficos, el desarrollo de la Neurociencia fue mucho más lento que el de áreas como la Bioquímica o la Biología molecular, que a mediados de los setenta ya tenían masa crítica suficiente como para contar con una asociación numerosa y activa como la Sociedad Española de Bioquímica o generar el Centro de Biología Molecular del CSIC. Posiblemente una de las causas más importantes del relativo retraso de la Neurociencia fueron sus dificultades y limitaciones metodológicas, agravadas por la situación precaria de la España 
de los setenta, y el poseer un lenguaje propio que dificultaba la comunicación con investigadores de otras áreas.

$\mathrm{Al}$ inicio de la década de los ochenta la investigación en Neurociencia en los paises desarrollados sufrió una eclosión que disparó un progreso acelerado que se ha mantenido hasta la actualidad. El impulso inicial se debió fundamentalmente a la incorporación de técnicas de la Biología molecular y celular a la investigación neurobiológica y a la aparición de una nueva Electrofisiología, más sencilla pero a la vez más analítica, gracias al descubrimiento de la técnica de «patch-clamp». Los estudios en preparaciones in vitro mostraron ser enormemente eficaces para potenciar el conocimiento de la estructura y función del tejido nervioso y facilitar el desarrollo de disciplinas como la Neurofarmacología o la Neuroquímica. Esta situación general afectó a la Neurociencia española de forma especial pues coincidió con la incorporación a nuestro país de neurobiólogos de la segunda generación, entrenados en laboratorios extranjeros, que contribuyeron decisivamente a la puesta al día de los grupos existentes y facilitaron el encuentro con investigadores formados en otras áreas pero atraídos por la investigación sobre el sistema nervioso. Una de las acciones que contribuyó de forma más decisiva a dar solidez al tejido de investigación en Neurobiología fue le creación de la Sociedad Española de Neurociencia (SENC) a la que además de neurohistólogos y neurofisiólogos, se incorporaron de forma particularmente activa neurofarmacólogos y neuroquímicos, así como biólogos del desarrollo y de las ciencias del comportamiento. La SENC apostó de forma rotunda por la modernidad y la calidad científica y en pocos años llegó a ser, y todavía es, una de las sociedades científicas nacionales más numerosas y de mayor prestigio. La potenciación de la Neurociencia española se manifestó por la modernización de Instituto Cajal del CSIC, al que se incorporaron numerosos investigadores de nivel internacional, la creación de nuevos institutos de Neurociencia, como el de Alicante, y la formación de grupos con calidad contrastada en centros del CSIC y las universidades en Barcelona, Madrid, Murcia, Valladolid, Santiago y Sevilla, entre otras. Aunque se han creado excelentes servicios de Neurología y Neurocirugía en los grandes hospitales que, en algún caso realizan una investigación de un nivel muy alto, en su conjunto la contribución de los grupos clínicos al desarrollo de la investigación ha sido inferior a lo deseable. Por diversas razones, los intentos de acercamiento de la investigación neurobiológica básica y clínica en la década de los setenta no llegaron a fructificar.

En la última década la Neurociencia española ha madurado gracias al fortalecimiento de los grupos ya existentes aunque no se ha estimulado 
la creación de grupos nuevos y no han aparecido iniciativas institucionales dignas de mención. Sin embargo, creo que no es exagerado afirmar que la Neurociencia es una de las áreas de la Biomedicina que más y mejor se ha desarrollado en nuestro país. Existe una investigación independiente, realizada en laboratorios españoles, con resultados que se publican en revistas del mayor nivel de la especialidad y es frecuente encontrar investigadores españoles con cargos de responsabilidad en comites internacionales, en consejos editoriales de revistas o participando como invitados en congresos de Neurociencia. A pesar de su calidad y del proceso de maduración reciente, hay que ser conscientes de que el tejido de la investigación neurobiológica en España, como en otras áreas, es frágil y elemental con un tamaño muy inferior a lo que cabría esperar de un país con el nivel de desarrollo socioeconómico de España. Como he comentado anteriormente, a la fragilidad y parquedad proverbial del sistema de ciencia y tecnología español hay que añadir, en el caso de la Neurociencia, la ausencia de plantilla administrativa en las universidades. En otras áreas, como la Bioquímica o la Fisiología, la existencia de profesores que son también investigadores, permite el que algunos grupos mantengan una cierta capacidad vegetativa de incorporación de nuevos profesionales.

\section{Perspectivas}

La comprensión de la organización y funcionamiento del sistema nervioso es uno de los límites de la ciencia moderna y un reto para la investigación de las próximas décadas. En los paises desarrollados la investigación en Neurociencia se considera prioritaria y de valor estratégico especial, no solo por la necesidad de prevenir o buscar remedios para las enfermedades neurodegenerativas o psiquiátricas, sino porque el cerebro es un máquina extraordinaria de procesado de la información cuyo conocimiento, además de ayudar a entendernos mejor, permitirá avances revolucionarios de las ciencias de la computación y la robótica. Por lo tanto, no basta con mantener o incluso potenciar la Neurociencia existente sino que ésta debe dar un salto cualitativo, sobre todo en lo que concierne a la creación de instituciones de excelencia, que faciliten la incorporación de España en condiciones creíbles y competitivas al concierto internacional.

Como indiqué en la «Introducción», aunque el mantenimiento de una visión integrada del cerebro es consustancial a la Neurociencia, los avances más importantes en Psicofarmacología o en Neurobiología 
molecular se han obtenido gracias al abordaje metodológico reduccionista. La realidad demuestra que para estudiar, por ejemplo, las causas de la degeneración neuronal o el efecto de los fármacos sobre los receptores sinápticos no se necesita el conocimiento detallado del funcionamiento de la corteza cerebral, que básicamente se desconoce. Reciprocamente, el investigador en Neurociencia cognitiva no tiene por qué ser experto en biofísica de canales iónicos. Por lo tanto, es posible que la forma más eficaz de enfocar el desarrollo institucional futuro de la Neurociencia sea basándose en grupos organizados en departamentos o unidades temáticas en las que se aborden problemas científicos concretos. Algunos de los temas que me parecen particularmente importantes son la degeneración, lesión y reparación en el sistema nervioso, la Neurobiología del desarrollo, la Neurobiología sensorial y, con relevancia especial, la Neurociencia cognitiva. Este último campo, practicamente inexistente en España, está teniendo un progreso reciente particularmente significativo debido al avance tecnológico en adquisición y procesado digital de imágenes. En mi opinión, la Neurociencia cognitiva debería ser objeto de un plan especial que facilitase su desarrollo en contacto con las ciencias de la conducta, la robótica, las matemáticas y las ciencias de la computación.

Una forma de potenciar la investigación en Neurociencia podría ser la creación de varias unidades temáticas ubicadas en institutos del CSIC o en centros mixtos CSIC/universidades que deberían estar coordinadas en redes nacionales. Otro modelo podría basarse en unidades asociadas a grandes centros hospitalarios donde la investigación básica y clínica se enfocasen hacia áreas temáticas específicas. Por ejemplo, sería enormemente ventajoso que las unidades de investigación sobre la degeneración, lesión y reparación del sistema nervioso se desarrollasen junto a departamentos de Neurología y Neurocirugía o las unidades de Neurociencia cognitiva junto a servicios de Radiología y Radiodiagnóstico. En mi opinión, una de las carencias más importantes de nuestro sistema de ciencia y tecnología es la no existencia de instituciones relacionadas con la investigación, el desarrollo y la innovación en Medicina. Esta situación es particularmente grave si se considera que contamos con un sistema sanitario público de tamaño considerable y de calidad asistencial más que aceptable.

En conclusión, la investigación en Neurociencia es de valor estratégico y debe ser prioritaria. El desarrollo reciente en este área ha sido importante pero queda mucho por hacer para que la investigación neurobiológica española tenga el tamaño, la estabilidad y la calidad requeridas para su homologación internacional. El avance de la in- 


\section{José López Barneo}

vestigación en Neurociencia podría basarse en unidades temáticas ubicadas en universidades y centros del CSIC. Una fórmula particularmente atractiva sería la creación de unidades de Neurociencia clínico-experimentales. 